\title{
Analyst
}

\section{Gold nanoparticle amplification strategies for multiplex SPRi- based immunosensing of human pancreatic islet hormones ${ }^{\dagger}$}

Received 00th January 20xx Accepted 00th January 20xx DOI: $10.1039 / x 0 x \times 00000 x$

www.rsc.org/

\author{
F. Rafael. Castiello and Maryam Tabrizian ${ }^{\mathrm{a}, \mathrm{b}}$
}

In this work, we demonstrate the potential use of SPRi for secretion-monitoring of pancreatic islets, a small micro-organ that regulates glucose homeostasis in the body. In the islets, somatostatin works as a paracrine inhibitor of insulin and glucagon secretion. However, this inhibitory effect is lost in diabetic individuals and little is known about its contribution to the pathology of diabetes. Thus, the simultaneous detection of insulin, glucagon and somatostatin, could help understand such communication. Previously, the authors introduced an SPRi biosensor to simultaneously monitor insulin, glucagon and somatostatin using an indirect competitive immunoassay. However, our sensor achieved a relatively high LOD for somatostatin detection ( $246 \mathrm{nM})$, the smallest of the three hormones. For this reason, the present work aimed to improve the performance of our SPRi biosensor using gold nanoparticles (GNPs) as a means of ensuring somatostatin detection from a small group of islets. Although GNPs amplification is frequently reported in the literature for individual detection of analytes using SPR, studies regarding the optimal strategy in a multiplexed SPR setup are missing. Therefore, with the aim of finding the optimal GNPs amplification strategies for multiplex sensing we compared three architectures: 1) GNPs immobilized on the sensor surface, 2) GNPs conjugated with primary antibodies (GNPs-Ab $b_{1}$ ) and 3) GNPs conjugated with a secondary antibody (GNPs-Ab $b_{2}$ ). Among these strategies an immunoassay using GNPs-Ab $b_{2}$ conjugates was able to achieve multiplex detection of the three hormones without cross-reactivity and with a LOD improvement of 9 fold for insulin, 10 fold for glucagon and $\mathbf{2 0 0}$ fold for somatostatin when compared to the SPRi biosensor without GNPs. The present work denotes the first report of the simultaneous detection of such hormones with a sensitivity level comparable to ELISA assays, particularly for somatostatin.

\section{Introduction}

Pancreatic islets are small micro-organ that regulates glucose homeostasis in the body ${ }^{1}$. Deficiencies in the islet's secretory pathways give rise to diabetes mellitus. However, little is understood about the paracrine communication occurring during glucose regulation ${ }^{1}$. For instances, it has been shown that somatostatin, secreted by the third most abundant cell type in the islets, is a potent paracrine inhibitor of both insulin and glucagon secretion ${ }^{2}, 3$. In a healthy adult, somatostatin secretion is normally stimulated by glucose, however this triggering effects are lost in type 1 and type 2 diabetes. This has been proposed to contribute to the impaired regulation of glucagon secretion in diabetes ${ }^{4,5}$. Thus, monitoring an islets' secretory fingerprint (SF) including the three most abundant secreting cells in the islet, could help to better understand such paracrine communications.

Pancreatic islet research up-to-date involves mostly the use of traditional bioassays for hormone quantification such as

\footnotetext{
a. Biomedical and Biological Engineering Department, McGill University, Montreal, QC, Canada.

b. Faculty of Dentistry, McGill University, Montreal, QC, Canada.

tElectronic Supplementary Information (ESI) available: Gold nanoparticle functionalization characterization: including surfaces and GNPs-Ab conjugates. See DOI: $10.1039 / x 0 x \times 00000 x$
}

ELISA ${ }^{6-8}$, patch clamp ${ }^{9-14}$, and capillary electrophoresis (CE) ${ }^{15-17}$. However, these techniques have a low throughput, are timeconsuming, labor extensive and can detect only one hormone at a time. Moreover, they face several challenges when attempting their implementation for multiplex analysis.

Most of these shortcomings could be addressed using optical, electrical, mechanical or magnetic biosensors ${ }^{18}$. Among these biosensing technologies, sensors based on the optical excitation of surface plasmons has increased in popularity in the last decade due to its simple, easy to use, non-invasive and label-free nature ${ }^{19}$. Surface plasmon resonance (SPR) biosensors present an additional advantage for the multiplex screening of biomolecular interactions when combine with imaging capabilities (SPRi) ${ }^{20}$. For instance, H. J. Lee et al. ${ }^{21}$ demonstrated the simultaneous detection of 3 low molecular weight protein biomarkers using SPRi, by creating a high-density antibody microarray achieving multiplex detection of the three protein markers down to $1 \mathrm{nM}$ concentrations. This makes SPRi a very desirable technique for secretion-monitoring of pancreatic islets.

In a recent work reported by the authors, a multiplex SPRibased biosensor was introduced as a viable tool for simultaneous quantification of insulin, glucagon, and somatostatin by performing three simultaneous competitive assays with monoclonal antibodies ${ }^{22}$. In this work an innovative surface chemistry was introduced and optimized for the 
detection of the three targeted peptides in a competitive immunoassay format with high antifouling properties, obtaining a limit of detection (LOD) of $1 \mathrm{nM}$ for insulin, $4 \mathrm{nM}$ for glucagon, and $246 \mathrm{nM}$ for somatostatin in multiplexed mode with a total analysis time of $\mathbf{2 1} \mathrm{min}$ per point. These LODs are satisfactory for the detection of insulin and glucagon as demonstrated by previous reports where these hormones were detected individually from secretions of a small group of islets within a microfluidic device ${ }^{15,16}$. However, there are no available reports regarding the required LOD for somatostatin within a similar islet population. Moreover, it is known that the number of somatostatin secreting cells within pancreatic islets is usually lower than that of insulin or glucagon secreting cells ${ }^{23}$.

Considering the important role of somatostatin in regulating insulin and glucagon secretion ${ }^{2}, 3$, and before moving to precious and scarce human islets studies, the aim of this work was to improve the performance of the previously developed SPRi biosensor to ensure that somatostatin secreted from a small group of islets can effectively, accurately and simultaneously be detected with other towed hormones.

Since its introduction by L. Lyon et al. ${ }^{24}$, gold nanoparticles (GNPs) have been the most commonly used method for improving the performance of SPR immunosensors. Two configurations are typically considered for the use of GNPs in SPR signal amplification: 1) the sensor surface modification with GNPs and 2) the labeling of a recognition element with GNPs. These strategies rely either on the coupling of local plasmon resonance of the GNP with the surface plasmon resonance of the system or in the increased mass attached to the recognition element for signal enhancement ${ }^{25}$, 26. Both GNPs amplification strategies have been frequently reported in the literature for individual detection of hormones such as insulin ${ }^{27}$, progesterone ${ }^{28}$, testosterone ${ }^{29}$ and other small analytes ${ }^{26,30,31}$, however, studies regarding the application of these strategies for multiplex hormones detection are scarce. Moreover, few reports exist regarding the use of GNPs amplification in a multiplex setting and they are focus on the detection of DNA sequences ${ }^{32}$ or cancer biomarkers by means of localized SPR using microscopy ${ }^{33}$. Although these reports demonstrated detection of target analytes in femtomolar levels in multiplex mode, there is a lack of formal studies regarding the optimal GNP amplification strategy for SPRi systems.

Thus, to establish the optimal signal amplification configuration for the multiplexed sensing of an islets' SF with SPRi, three GNPs amplification strategies were investigated including 1) GNPs immobilized on the sensor surface, 2) GNPs conjugate with primary antibodies (GNPs-Ab 1 ) and 3) GNPs conjugated with a secondary antibody $\left(G N P s-A b_{2}\right)$. For this study, somatostatin was used as the 'reference' hormone, as it is the smallest among the three islets' SF, to first test the performance of the aforementioned SPR signal amplification strategies in an indirect competitive assay. Then, the biosensor performance was assessed in multiplex mode to determine the LOD and dynamic range for the three targeted hormones simultaneously.

\section{Experimental section}

\section{Materials and methods}

$20 \mathrm{~nm}$ gold nanoparticles (GNPs) in citrate buffer, ethanolamine hydrochloride, N-Hydroxysuccinimide (NHS), N-(3Dimethylaminopropyl)- $\mathrm{N}^{\prime}$-ethylcarbodiimide hydrochloride (EDC), hexa(ethylene glycol) dithiol (HEGD), bovine serum albumin (BSA), hydrochloric acid $(\mathrm{HCl})$, glycerol, sodium hydroxide $(\mathrm{NaOH})$, human glucagon and human somatostatin were purchased from Sigma-Aldrich (St. Louis, MO, USA). Absolute ethanol was purchased from Fisher Scientific (Fair Lawn, NJ, USA), phosphate-buffered saline (PBS) tablets, tween 20 and glycine were purchased from BioShop Canada Inc. (Burlington, Ontario, Canada). (3,3'-dithiobis(sulfosuccinimidyl propionate)) (DTSSP) was purchased form Thermofisher Scientific. Tris-buffered saline (TBS) with $1 \%$ casein, goat antimouse IgG1and goat anti-rat IgG2a secondary polyclonal antibodies were purchased from BIO-RAD. Anti-insulin antibody $(6.2 \mathrm{mg} / \mathrm{mL})$ and human insulin were purchased from PROSPECT (Ness, Ziona, Israel). Anti-somatostatin monoclonal antibodies $(200 \mu \mathrm{g} / \mathrm{mL}$ each) were purchased from Santa Cruz Biotechnologies, Inc. (Mississauga, ON, Canada). Anti-glucagon monoclonal antibodies were purchased from Abcam (Cambridge, MA, USA). $\mathrm{CH}_{3} \mathrm{O}-\mathrm{PEG}-\mathrm{SH}$ (MW $1200 \mathrm{Da}$ ) was purchased from Rapp Polymers GmbH (Tübingen, Germany). 16-mercaptohexadecanoic acid (MHDA) from Prochimia Surfaces Sp. (Zacisze, Sopot, Poland). Borate buffer $0.5 \mathrm{M} \mathrm{pH} 8.5$ was purchase was purchased from Alfa-Aesar (USA).

\section{Substrate preparation}

Cleaned microscope glass slides $(12 \mathrm{~mm} \times 25 \mathrm{~mm} \times 1 \mathrm{~mm}, \mathrm{n}=$ 1.518) substrates were coated with $2 \mathrm{~nm} \mathrm{Cr}$ as an adhesion layer, followed by the deposition of a thin Au layer of $48 \mathrm{~nm}$ using E-beam vapor deposition under high vacuum. The slides were then coupled to an SF11 equilateral triangular prism (nSF$11=1.765)$ using a refractive index matching liquid. Goldcoated prisms ( $n=1.765$ ) were purchased from Horiba Scientific, France and used as received.

\section{SPRi measurements}

SPRi detection was performed using a scanning-angle SPRi instrument (model SPRi Lab+, Horiba, France). The SPRi apparatus is equipped with an $800 \mathrm{~nm}$ LED source, a CCD camera, and a microfluidic flow cell. All experiments were performed at $25{ }^{\circ} \mathrm{C}$ by keeping the instrument inside an incubator (Memmert Peltier, Rose Scientific, Canada).

To select the working angle for kinetic analysis, the slope of the plasmon curves was computed automatically by the instrument's software. The selected angle corresponds to the point of the plasmon curve at which the slope was maximum. Reflectivity shift $(\Delta R(\%))$ was measured upon stabilization of the baseline. After each analyte injection, the substrate was rinsed with running buffer PBS-T (PBS with $0.002 \%$ tween 20), and the $\Delta R$ was calculated by the difference between the buffer signal before and after the analyte injection. The signal was recorded at least on three spots for each analyte and controls 


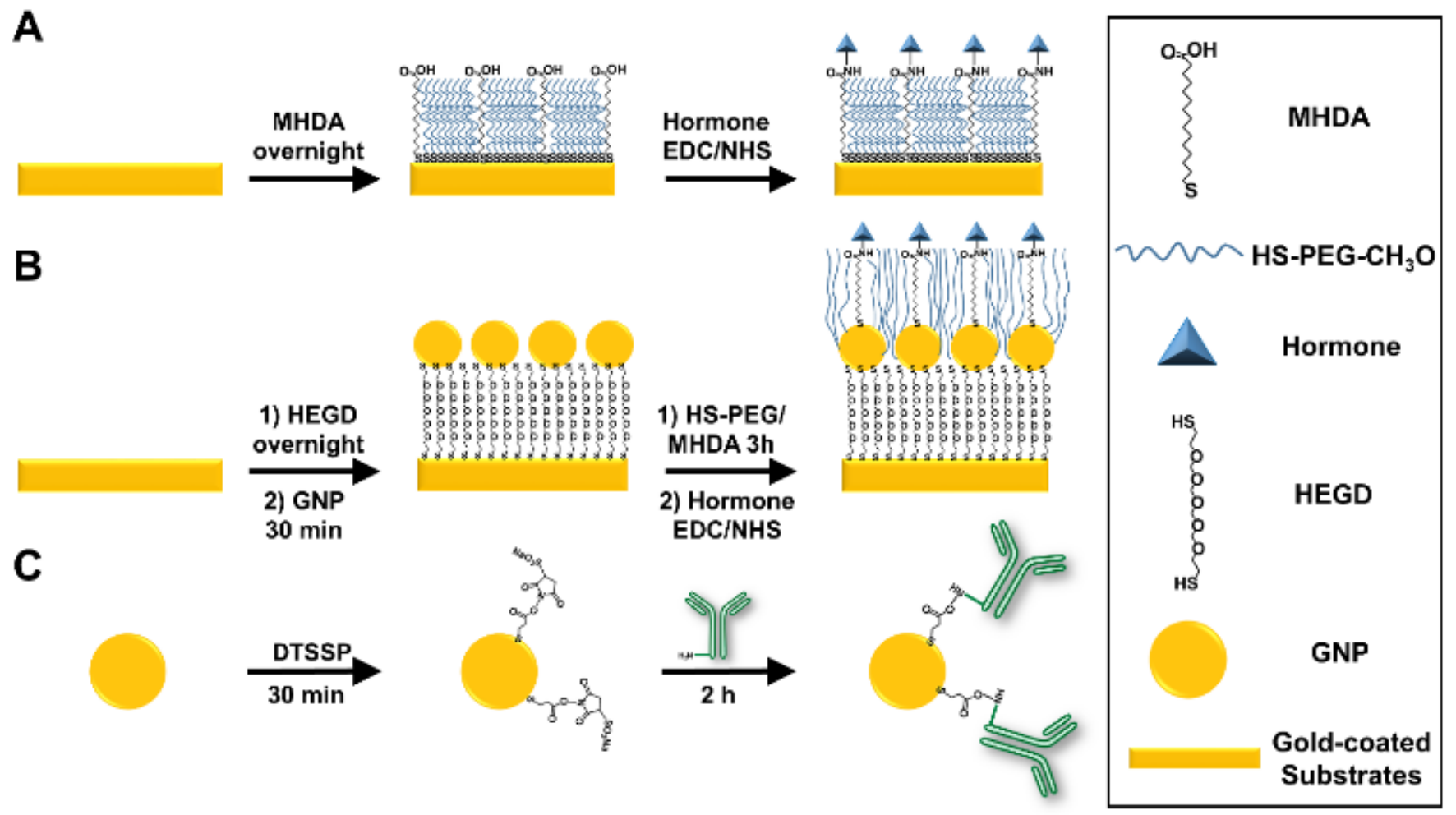

Fig. 1 Schematic representation of the typical surface functionalization of: A) Gold-coated substrates functionalized with a self-assembled monolayer (SAM) of 16mercaptohexadecanoic acid (MHDA)/CH3O-PEG-SH (PEG) and subsequently with the targeted hormone, B) gold-coated substrate surface functionalized with a SAM of hexa(ethylene glycol) dithiol (HEGD), GNPs and subsequently with the targeted hormone and C) covalent antibody functionalization of for the formation of GNPs-conjugates using (3,3'dithiobis(sulfosuccinimidyl propionate)) (DTSSP).

to determine the average $\Delta \mathrm{R}$ values. All experiments were performed using an injection loop with a fixed volume of $200 \mu \mathrm{L}$ and a constant flow rate of $20 \mu \mathrm{L} / \mathrm{min}$, with the exception of functionalization steps where the flow rate was adjusted depending on the required contact time.

All SPR plots are presented as a function of reflectivity shift $(\Delta \mathrm{R}(\%))$ vs time. A Savitzky-Golay smoothing polynomial function of second order was applied to all plots using OriginLab 2018 (b.9.5.5.409).

\section{Surface immobilization of hormones}

Following a protocol previously developed by authors ${ }^{22}$, an ethanolic solution of $0.5 \mathrm{mM} \mathrm{CH}_{3} \mathrm{O}-\mathrm{PEG}-\mathrm{SH}$ and $0.5 \mathrm{mM}$ MHDA were prepared and mixed at a molar ratio of $40 \%$ MHDA and $60 \%$ PEG (60-PEG/40-MHDA). Gold-coated prisms and slides were immersed in the ethanolic solution overnight to allow selfassembly monolayer (SAM) formation. Finally, they were thoroughly rinsed with absolute ethanol, DI water and dried under a stream of $\mathrm{N}_{2}$.

For individual somatostatin sensing experiments, SAMfunctionalized slides were placed on the SPRi system for subsequent functionalization. An initial conditioning step was performed by four serial injections of a 1M glycine $\mathrm{pH} 2.5$ (1MGly) solution (contact time of 2 min each). Then, PBS-T was allowed to run until a stable baseline was obtained. Next, covalent immobilization of insulin, glucagon or somatostatin via NHS/EDC chemistry was performed following Gobi et al. protocol ${ }^{34}$. Briefly, an aqueous solution containing $2 \mathrm{mg} / \mathrm{mL}$
NHS, $2 \mathrm{mg} / \mathrm{mL} E D C$ and $50 \mu \mathrm{g} / \mathrm{mL}$ of the desired hormone was injected into the system with a contact time of $1 \mathrm{~h}$. Next, an injection of $1 \mathrm{M}$ ethanolamine hydrochloride $\mathrm{pH} 8.5$ (contact time $10 \mathrm{~min}$ ) was performed to inactivate any unfunctionalized $-\mathrm{COOH}$ groups. Then, two serial injections of $1 \mathrm{M}-\mathrm{Gly}$ solution (contact time $1 \mathrm{~min}$ each) were performed to remove weakly bound hormones. Finally, a blocking solution containing 5\% BSA and $1 \%$ casein in TBS buffer was injected (contact time of 30 min) and subsequently, at least 3 injections of the $1 \mathrm{M}$-Gly solution were made to remove weakly bound proteins. Figure 1A shows a schematic representation of a typical surface hormone functionalization.

For multiplex measurements, gold-coated prisms were functionalized using the procedure described for glass slides outside of the SPR system. After conditioning, four individual solutions containing NHS/EDC and each targeted hormone (insulin, glucagon, somatostatin) or a control (BSA) were spotted $(150 \mathrm{~nL})$ in triplicate on the prisms and incubated in a humidity chamber for $1 \mathrm{~h}$. After incubation, the prisms were rinsed with DI water and exposed to $1 \mathrm{M}$ ethanolamine hydrochloride $\mathrm{pH} 8.5$ for $10 \mathrm{~min}$. Next, the prisms were exposed to the blocking solution for $30 \mathrm{~min}$ and subsequently, rinsed with PBS-T. Finally, the prisms were placed in the SPR system and a $1 \mathrm{M}$-Gly solution was injected at least three times to obtain a stable baseline before starting with the experiments.

Immobilization of GNPs on the gold-coated sensor's surface 
In this work, $20 \mathrm{~nm}$ commercial GNP were selected since it has been reported in literature as an optimal size for SPR amplification when either immobilized in the sensor's surface or for antibody modified GNPs immunoassays of small molecules $28,35,36$.

The immobilization of commercial $20 \mathrm{~nm}$ GNPs on the goldcoated sensor's surface was achieved according to a protocol developed by Taufik et al. ${ }^{37}$ with minor modifications. After cleaning, gold-coated slides were immersed in an ethanolic solution of $2 \mathrm{mM}$ HEGD overnight to allow SAM formation. After rinsing with ethanol and DI water, the slides were exposed to an aqueous solution containing $20 \mathrm{~nm}$ GNPs $\left(O_{520}=1.0\right)$ and incubated for one hour. Next, the slides were rinsed with DI water and placed in a 60-PEG/40-MHDA ethanolic solution for 3 hours. Finally, hormone functionalization was performed according to the procedure described in the previous section. Figure 1B shows a schematic representation of the surface functionalization with GNPs and hormones.

\section{Antibody-GNP functionalization}

The functionalization protocol for commercial $20 \mathrm{~nm}$ GNPs $\left(O D_{520}=1\right)$ with primary and secondary antibodies was adapted from a previous report by J. D. Driskel et al. ${ }^{38}$ using DTSSP as a bifunctional crosslinker. Briefly, $134 \mu \mathrm{L}$ of $50 \mathrm{mM}$ borate buffer $\mathrm{pH} 8.5$ was added to a $1 \mathrm{~mL}$ suspension of $20 \mathrm{~nm}$ GNP to adjust the $\mathrm{pH}$. Next, $26 \mu \mathrm{L}$ of $20 \mu \mathrm{M}$ DTSSP was added to the GNPs and incubated for $30 \mathrm{~min}$ to form a thiolate monolayer through cleavage of the DTSSP disulfide bond. The suspension was then centrifuged at $17,500 \mathrm{~g}$ for $30 \mathrm{~min}$. Then, the supernatant containing excess DTSSP was removed and the GNPs were resuspended in $1 \mathrm{~mL}$ of $2 \mathrm{mM}$ borate buffer. Immediately after, $20 \mu \mathrm{g}$ of the desired antibody was added to the DTSSP-GNPS suspension and incubated for 1.5 hours. The suspension was then centrifuged at 17,500g for $30 \mathrm{~min}$, the supernatant was removed, and the GNPs were resuspended in $1 \mathrm{~mL}$ of $2 \mathrm{mM}$ borate buffer containing $1 \%(\mathrm{~m} / \mathrm{v}) \mathrm{BSA}$ and incubated for $30 \mathrm{~min}$ to allow the BSA to block any unreacted DTSSP and nonspecific binding sites. The centrifugation/resuspension cycle was repeated two additional times using $2 \mathrm{mM}$ borate buffer for resuspension to remove excess antibody and BSA. The final volume of the solution after the centrifugation/resuspension cycles was fixed to approximately $200 \mu \mathrm{L}\left(\mathrm{OD}_{525} \approx 4\right)$ and the solution was stored at $4{ }^{\circ} \mathrm{C}$. The functionalized GNPs were diluted to the desired concentration in PBS-T prior to use in immunoassays. Figure $1 \mathrm{C}$ shows a schematic representation of antibody-GNP functionalization. Successful GNPs functionalization for all antibodies was confirmed by the shift in absorbance maxima of the GNPs from 520 to 525 nm (Fig. S2 provided in supporting information).

\section{Competitive immunoassays}

The four configurations used for indirect competitive immunoassays consisted in a surface with (Fig. 2A) and without GNPs (Fig. 2B), primary antibodies GNPs (GNP-A $b_{1}$ ) conjugates (Fig. 2C) and secondary antibodies GNPs (GNP-A $b_{2}$ ) conjugates (Fig. 2D). Since somatostatin was the smallest of the targeted

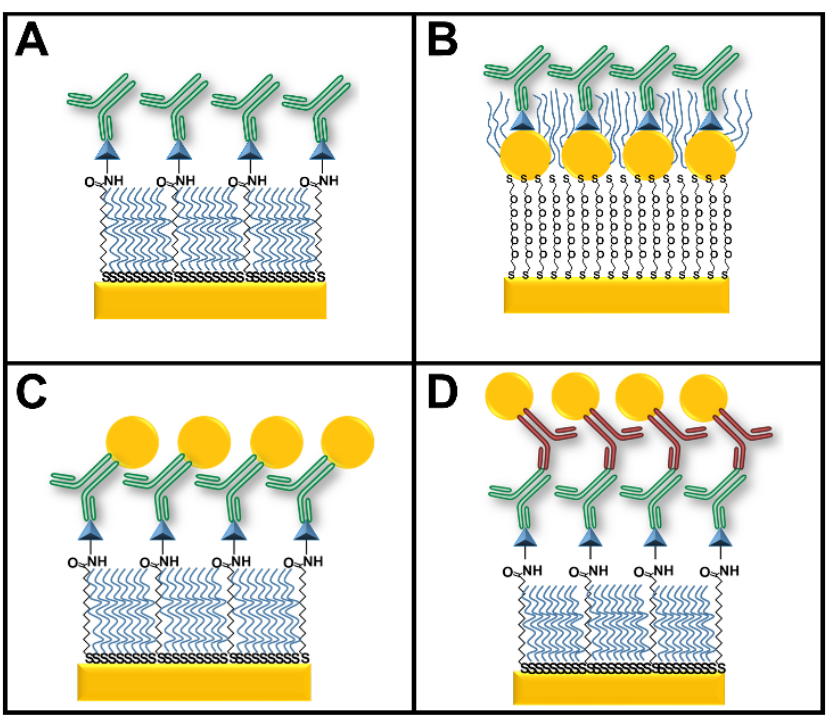

Fig. 2 Schematic representation of the four configurations of competitive immunoassay used for this work: A) an assay involving only primary antibodies and the hormone immobilized on a gold surface used as control, B) an assay where GNPs are present on the surface and the hormone is immobilized on top of the GNPs, C) an assay where GNPs are conjugated with monoclonal primary antibodies (GNPs-A $b_{1}$ ) and D) an assay involving amplification using GNPs conjugated with a polyclonal secondary antibody (GNPs-Ab $b_{2}$ ) after a primary competitive assay.

hormones, it was used as the "reference" hormone in all individual hormone assays. The optimal primary antisomatostatin antibody, GNPs-Ab1 and GNPs-Ab2 concentration were defined as the concentration that could generate a small but detectable SPR signal of $\Delta R \approx 1$, previously reported as a reliable $\Delta R$ for this type of assays ${ }^{39}$.

Standard somatostatin solutions were prepared by serial dilution in PBS-T buffer with concentrations ranging between $0.01-4000 \mathrm{ng} / \mathrm{mL}$. These solutions were then mixed with 2 $\mu \mathrm{g} / \mathrm{mL}$ of anti-somatostatin antibody for assays involving only primary antibodies, $0.6 \mu \mathrm{g} / \mathrm{mL}$ of antibody for the assay with GNP-A $b_{2}$ conjugates or a 1:50 dilution (from $\mathrm{OD}_{525} \approx 4$ ) GNP-Ab 1 conjugates. All mixtures were incubated for 2 min with gently mixing by inverting upside down and then injected into the system from highest to lowest hormone concentration (contact time of $10 \mathrm{~min}$ ) starting with a blank solution containing only anti-somatostatin antibodies or GNP-Ab $b_{1}$ conjugates. For secondary antibody conjugates assays, immediately after antisomatostatin antibody injection, GNPs-Ab 2 conjugates were injected (1:50 dilution from $\left.\mathrm{OD}_{525} \approx 4\right)$. The optimal primary anti-somatostatin antibody, GNPs-Ab $b_{1}$, and GNPs-Ab concentration were defined as the concentration that could generate a small but detectable SPR signal of $\Delta R \approx 1$, previously reported as a reliable $\Delta R$ for this type of assays ${ }^{39}$.

During calibration curve experiments, different sensor regeneration solutions were tested including $10-50 \mathrm{mM} \mathrm{NaOH}$, $50 \mathrm{mM} \mathrm{NaOH}$ 5-50\% (v/v)-glycerol, 0.1-1 M glycine ( $\mathrm{pH} 1.5)$, 0.1-1 M glycine ( $\mathrm{pH} 1.5)$ with $1 \%(\mathrm{v} / \mathrm{v}) \mathrm{DMSO}$, and $2 \mathrm{M} \mathrm{MgCl}$. From these solutions, $50 \mathrm{mM} \mathrm{NaOH}$ with $25 \%$ glycerol provided the most efficient conditions for regeneration and it was used throughout all the experiments.

For multiplex assays, standard solutions having a mix of insulin, glucagon, and somatostatin were prepared in PBS-T 
buffer at a concentration range of $0.01-4000 \mathrm{ng} / \mathrm{mL}$ and mixed with a cocktail of primary antibodies containing anti-insulin $(0.2$ $\mu \mathrm{g} / \mathrm{mL})$, anti-glucagon $(0.05 \mu \mathrm{g} / \mathrm{mL})$ and anti-somatostatin $(0.6$ $\mu \mathrm{g} / \mathrm{mL}$ ). Similar to individual somatostatin assays, the mixtures were incubated for $2 \mathrm{~min}$ with gentle mixing and serially injected over the spotted sensor chip from highest to lowest hormone concentration (contact time of $10 \mathrm{~min}$ ) starting with a blank solution containing only the antibody cocktail. Subsequently, a GNPs-A $\mathrm{b}_{2}$ mixture containing GNPs conjugated to anti-mouse IgG1 (1:100 dilution from $\mathrm{OD}_{525} \approx 4$ ) and anti-rat Ig2a ( $1: 50$ dilution from $O_{525} \approx 4$ ) was injected into the system. Each sensing cycle comprised: hormone primary antibody mixing and incubation for $2 \mathrm{~min}$, cocktail injection for $10 \mathrm{~min}, 3$ min buffer washing, 10 min injection of a GNPs-Ab $b_{2}$ mixture and 2 injections of regeneration solution with a contact time of $30 \mathrm{~s}$ with 3 min washing with buffer in-between.

\section{Statistics}

Relative binding $\left(\mathrm{C} / \mathrm{C}_{0}\right)$ was calculated for all competitive immunoassays, by dividing the response of the standard solutions containing hormones $(\mathrm{C})$ by the response of the blank solution containing only a fixed concentration of antibodies $\left(C_{0}\right)$. To generate calibration curves $C / C_{0}$ was plotted against hormone concentration. The calibration curves were fitted using a non-linear 4 parameter logistic (4PL) model using OriginLab 2018 (b.9.5.5.409). The LOD for all immunoassays was calculated from the calibration curves as the blank signal $\left(C_{0}\right)$ minus three times the standard deviation. The dynamic range for the competitive immunoassay was established between 0.2 $\mathrm{C} / \mathrm{C}_{0}$ and $0.8 \mathrm{C} / \mathrm{C}_{0}$. All data are expressed as the average of at least 3 independent experiments \pm standard deviation (SD).

\section{Results and discussion}

\section{GNP amplification strategies comparison for competitive immunoassays}

First, the formation of a chemically-linked layer of GNPs was performed using a SAM of a dithiol alkane (HEGD). HEGD allowed anchoring of the GNPs to the gold-coated sensor's surface through the thiol group on each end of the molecule ${ }^{37}$. AFM analysis indicated the successful immobilization of the GNPs by a significant change in surface morphology from a clean gold surface to a GNPs-modified surface as clearly observed in Figure S1 provided in supporting information. This was further confirmed by a change in surface RMS roughness from $0.68 \mathrm{~nm}$ to $2.41 \mathrm{~nm}$ and later to $5.36 \mathrm{~nm}$ from a clean surface to a SAM-functionalized surface, and to a GNPsfunctionalized surface. The signal amplification rationale here is that the activation of the GNPs localized SPR due to the proximity of the immobilized GNPs to the sensor's surface can lead to different resonance properties of the overall SPR system with additional resonance shifts, resulting in an enhanced sensitivity of the biosensor ${ }^{25}$.

For all GNPs-antibody conjugates, functionalization was confirmed by a shift observed in the maximum absorption peak from 520 to $525 \mathrm{~nm}$ (Fig. S2 in supporting information). In the
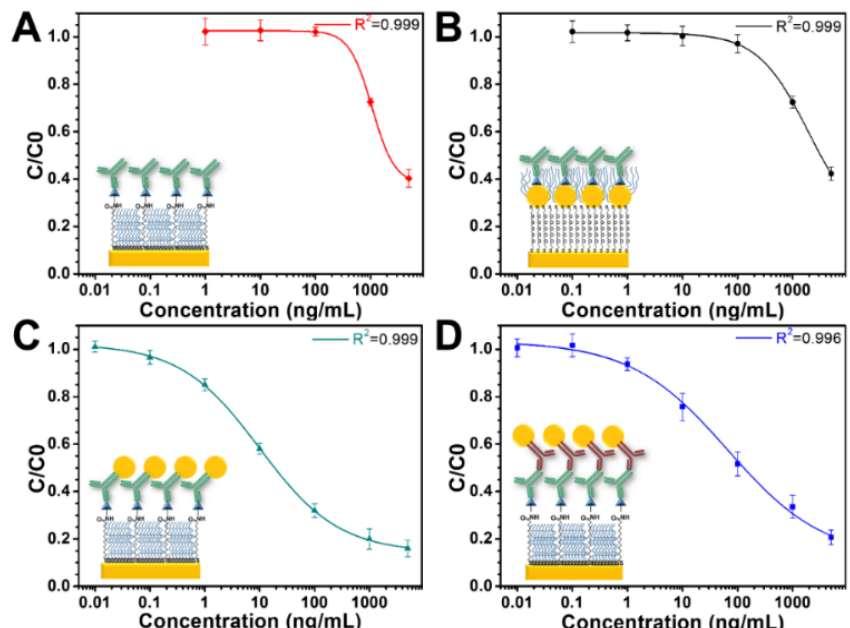

Fig. 3 Somatostatin sensing calibration curves in PBS-T for competitive immunoassays involving A) only primary antibodies, B) GNPs on the sensor's surface, C) GNPs conjugated with monoclonal primary antibodies (GNPs-A $b_{1}$ ) and $D$ ) an assay with GNPs conjugated with a polyclonal secondary antibody $\left(G N P s-A b_{2}\right)$. Solid lines correspond to the fitting of a nonlinear 4PL model. Error bars represent the standard deviation from 3 independent experiments $(n=3)$.

case of GNPs-Ab $b_{1}$ conjugates, the rationale behind this strategy is that the increased mass of the antibody due to the linked GNPs will result in a higher refractive index change on the SPR surface, thereby producing a larger SPR shift ${ }^{26}$. Additionally, due to the close proximity of the GNPs to the SPR surface $(<15 \mathrm{~nm})$ signal enhancement is also linked to electromagnetic field coupling between surface SPR and the GNPs localized SPR ${ }^{26}$. Regarding the use of GNPs-Ab $b_{2}$ conjugates, the signal amplification is only expected from the increased mass of the antibody due to the linked GNPs, as the GNPs are quite far from the surface 26

Figure 3 shows the sensor calibration curves for somatostatin detection where the mean relative binding values $\left(C / C_{0}\right)$ were plotted as a function of hormone concentration for all sensing strategies. For these experiments, detection without GNPs was used as a control assay (Fig. 3A). Additionally, the entire sensor surface was functionalized with somatostatin, and the SPR shift for all sensing events was obtained as the mean of at least 10 spots from different regions of the chip. Then, an average of $\Delta R$ of three independently prepared chips was calculated representing their corresponding standard deviation (SD). The LOD and dynamic range for each sensing strategy are shown in Table 1.

Noteworthy, the concentration of the control assay (Fig. 3A) and the strategy using a GNPs-modified surface (Fig. 3B) was set to $2 \mu \mathrm{g} / \mathrm{mL}$ to facilitate comparison and to easily observe SPR signal enhancement. Interestingly, these two sensing strategies presented similar LODs. However, the GNPs-modified surface showed a higher SPR signal (Fig. S3 in supporting information) compared to the signal obtained for the surface without GNPs as clearly observable in the $\Delta \mathrm{R}(\%)\left(\mathrm{C}_{0}\right)$ values in Table 1 . Indeed, this seems consistent with recent reports where the LOD of the calibration curve of an indirect competitive immunoassay using GNP-modified sensors did not change even when the SPR signal was enhanced 31, 40. A possible explanation is that the performance of an indirect competitive immunoassay highly 

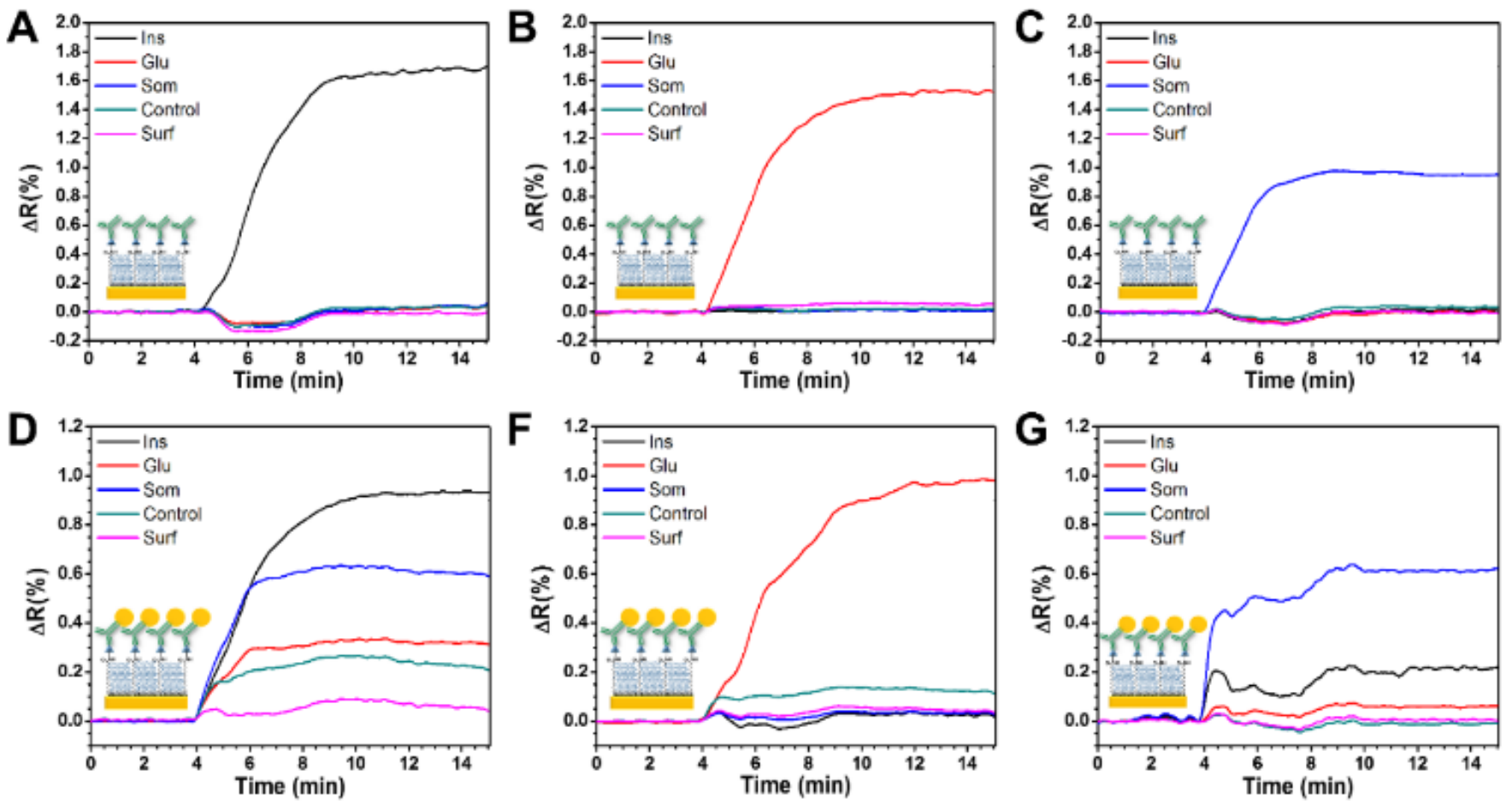

Fig. 4 Comparison of the sensor specificity in multiplex mode multiplex. The first row shows the specificity of the sensor without signal amplification for A) anti-insulin, B) antiglucagon, and C) anti-somatostatin. Second row shows the specificity of the sensor on the same surface with signal amplification for D) GNPs-anti-insulin, F) GNPs-anti-glucagon and G) GNPs-anti-somatostatin. Immobilized BSA ("Control") and the bare SAM surface ("Surf") were used as negative controls.

depends on the affinity constant of the immunoreaction ${ }^{31}$. Therefore, only strategies affecting the affinity of the antigenantibody system such as the GNPs-Ab conjugation could improve the LOD of the SPR sensor ${ }^{31}$.

Finally, from all immunosensing strategies, the competitive immunoassay using GNPs-Ab 1 conjugates presented the best performance decreasing the LOD three orders of magnitude compared to the control assay from $450 \mathrm{ng} / \mathrm{mL}$ to $240 \mathrm{pg} / \mathrm{mL}$. Hence, this immunoassay amplification strategy was selected for further development of the multiplex hormone-sensing assay.

Table 1 SPR Sensing Performance for somatostatin using different immunosensing strategies

\begin{tabular}{ccccc}
\hline Strategy & $\Delta \mathrm{R}(\%)\left(\mathrm{C}_{0}\right)$ & $\begin{array}{c}\text { LOD } \\
{[\mathrm{ng} / \mathrm{mL}]}\end{array}$ & $\begin{array}{c}\text { LOD } \\
{[\mathrm{nM}]}\end{array}$ & $\begin{array}{c}\text { Dynamic } \\
\text { Range } \\
{[\mathrm{ng} / \mathrm{mL}]}\end{array}$ \\
\hline $\begin{array}{c}\text { Primary } \\
\text { antibodies }\end{array}$ & $1.10 \pm 0.03$ & 450 & 275 & $754-4000^{\mathrm{a}}$ \\
GNPs on surface & $1.72 \pm 0.05$ & 404 & 247 & $626-4000^{\mathrm{a}}$ \\
GNPs-Ab & $1.26 \pm 0.02$ & 0.24 & 0.15 & $1.54-780$ \\
GNPs-Ab & $0.93 \pm 0.04$ & 1.75 & 1.07 & $7.5-4000^{\mathrm{a}}$
\end{tabular}

a Highest concentration tested.

\section{GNP-amplified multiplex hormone sensing}

Fig. 4A-C shows the assessment of cross-reactivity for primary antibodies. Individual injection of each antibody caused an increase in SPR signal only on its relevant spot, demonstrating specificity and low cross-contamination between spots.
Moreover, there was a negligible response on the control spots, indicating good antifouling properties.

However, when a similar experiment was performed using GNPs-Ab ${ }_{1}$ conjugates, high cross-reactivity was observed. This effect was particularly large for GNPs-anti-insulin conjugates which generated a non-specific signal increase in all the functionalized spots including the two negatives controls (Fig. 4D). When GNPs-anti-glucagon conjugates were injected, crossreactivity with insulin and somatostatin spots was not observed, however some degree of non-specific interactions were detected for the negative control spots (Fig. 4F). For GNPs-antisomatostatin conjugates, cross-reactivity was also observed mostly with insulin spots (Fig. 4G). Additional antibodies from different species and companies were used for GNPs-Ab G $_{1}$ conjugates for anti-insulin and anti-somatostatin. However cross-reactivity was always present (data not shown). This has been reported in literature as a recurring problem for multiplex immunoassays ${ }^{41,} 42$ particularly for GNP conjugates since it has been shown that such conjugations can modify the activity of the antibodies. In theory, a combination of GNPs-Ab conjugates with little or null cross-reactivity for our system could be achieved, however testing a library of antibodies would be time consuming and cost-ineffective. Due to this dilemma, the second-best amplification strategy (GNP-Ab $\left.{ }_{2}\right)$ was selected for further development of our multiplex immunosensor. For this strategy, some degree of crossreactivity could occur since the secondary antibodies are similarly conjugated to GNPs. In general, antibody conjugation is known to affect the antibody's affinity ${ }^{31}$, however this did not seem to hinder the possibility for multiplex sensing as later demonstrate in this section. 

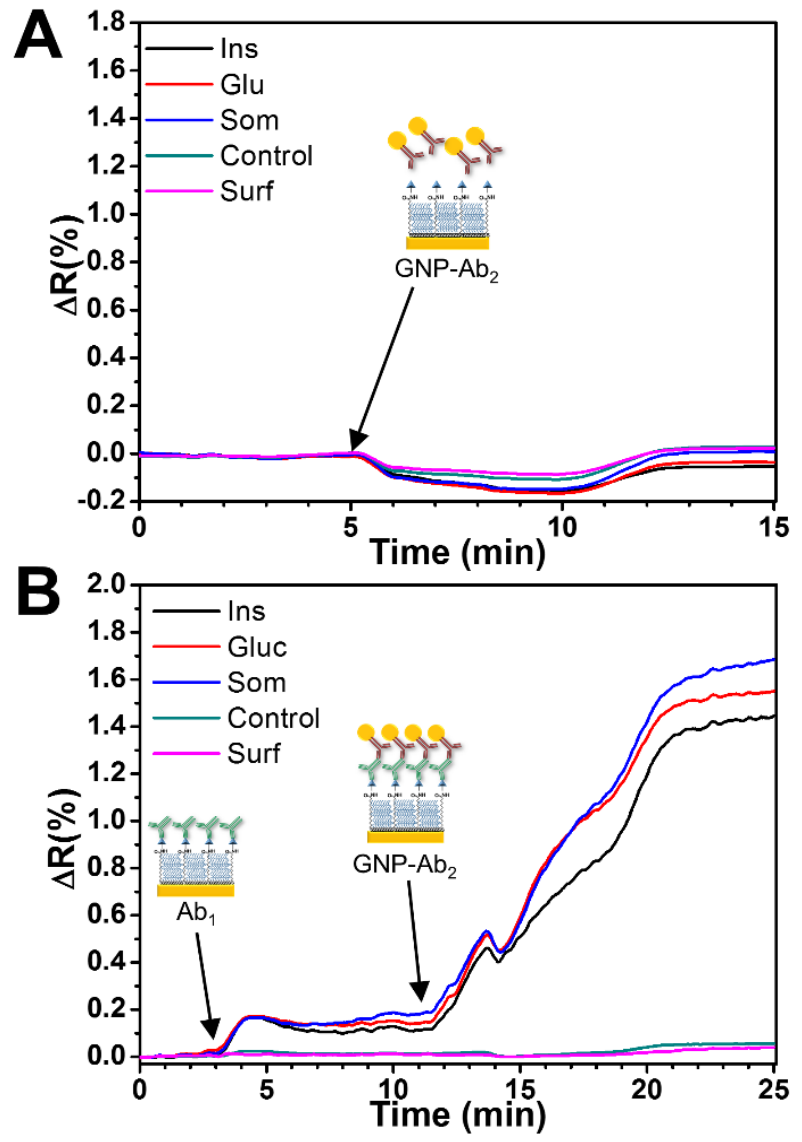

Fig. 5 Real-time SPR angle shift sensorgrams of A) the nonspecific binding effect of GNPs-A $b_{2}$ conjugates and $\mathbf{B}$ ) the specific binding effect of GNPs-A $b_{2}$ conjugates after primary antibody injection (blank injection $\mathrm{C}_{0}$ ). Immobilized BSA and the bare SAM surface identified as "Control" and "Surf" were used as negative controls.

The nonspecific binding effect of the GNPs-Ab ${ }_{2}$ conjugates on the analyte spots was determined prior to the multiplex assay through the injection of a mix of GNPs-goat anti-mouse IgG1and GNPs-goat anti-rat IgG2a over the sensor surface. As seen in Figure 5A, a minimum SPR angle shift was detected during the injection with the signal returning to similar baseline levels after a few minutes of PBS-T washing, indicating a negligible nonspecific binding effect of the GNPs-A $b_{2}$ conjugates to the different surface spots.

Figure 5B shows a typical sensor response to a blank solution (mix of all antibodies) and the subsequent amplification effect of the GNPs-Ab $b_{2}$ conjugates. First, the injection caused a small increase in SPR signal on the relevant hormone spots while an almost no response for the control spots, indicating specific binding. For these experiments, the initial concentration of primary antibodies $\left(C_{0}\right)$ was fixed to 0.2 $\mu \mathrm{g} / \mathrm{mL}$ for anti-insulin, $0.05 \mu \mathrm{g} / \mathrm{mL}$ for anti-glucagon and 0.6 $\mu \mathrm{g} / \mathrm{mL}$ for anti-somatostatin. This $A b_{1}$ concentration produced a small signal of $\approx 0.15 \Delta R$ for all hormone spots. The subsequent injection of GNPs-Ab $b_{2}$ conjugates generated an SPR signal enhancement of $\approx 10$ times, which was consistent with previous literature reports where $20 \mathrm{~nm}$ GNPs-Ab 2 conjugates have been used for signal amplification 28,43 .

It is noteworthy the fact that the $C_{0}$ signal obtained for somatostatin during individual GNPs-Ab $b_{2}$ was smaller $(\Delta R=$

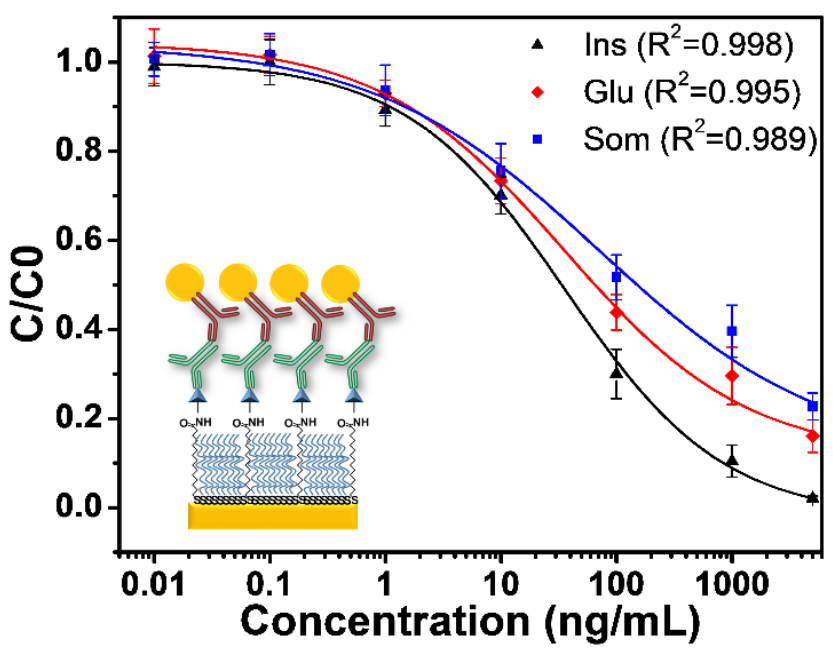

Fig. 1. Calibration curves for a multiplex immunoassay using GNPs-Ab $\mathrm{B}_{2}$ conjugates as amplification strategy in PBS-T. Solid lines correspond to the fitting of a nonlinear 4PL model for different concentration of insulin (blue), glucagon (red), and somatostatin (black). For each hormone, mean relative binding values $\left(C / C_{0}\right)$ were plotted as a function of hormone concentration $(\mathrm{ng} / \mathrm{mL})$. The mean SPR shift was measured in sets of triplicate spots for each hormone and the controls. Then, the average SPR shift was calculated using 3 independent multiplex sensors $(n=3)$ corresponding to the reported SD.

0.93, Table 1) compared to that obtained during the multiplex assay $(\Delta R=1.51$, Table 2$)$. This difference in likely due to some cross-reactivity between the different species of secondary antibodies in the GNPs-Ab $b_{2}$. However, due to the absence of non-specific interactions with the hormone spots and the controls, it was possible to use these conjugates for multiplex detection since the sensor response $(\Delta R)$ was always consistent and reproducible for all targeted hormones.

To further assess the sensor's resistance to non-specific adsorption of proteins, at the end of each calibration curve two solutions containing either BSA or LYZ, both with a final concentration of $1 \mathrm{mg} / \mathrm{mL}$ was injected to the system following the same protocol as for the hormone immunoassay. After an abruptly increased in the SPR signal during the injections due to the high bulk refractive index of the solution, the shift in reflectivity was measured after 10 minutes of contact time and 5 minutes of PBS-T wash. Since LYZ presented the largest $\Delta R$ among the two tested proteins, it was used as reference to quantitatively evaluate the antifouling properties of the sensor. The mean $(n=3) \triangle R$ response to BSA and LYZ from the hormonefunctionalized spots and controls is showed in Table S1 in supporting information. It was found that the adsorbed amount of $L Y Z$ was $\approx 100 \mathrm{pg} / \mathrm{mm}^{2}$ for all spots assuming that $1 \mathrm{RU}=1 \mathrm{RIU}$ $=1 \mathrm{pg} / \mathrm{mm}^{2}$ of surface mass shift for a fixed wavelength of 800 $\mathrm{nm}^{44}$. This was very consistent with the definition of an antifouling surface ${ }^{45}$. Moreover, a short (30 s) injection of regeneration solution returned the baseline to its original value, indicating a weak interaction of LYZ and BSA on the sensor's surface.

Figure 6 shows the average calibration curves for the multiplex sensing of serially diluted mixtures of insulin, glucagon, and somatostatin in PBS-T obtained from three independent sensor chips. The calculated LOD and dynamic 
range obtained (Table 2 ) for the three hormones in multiplexed mode were somehow comparable to that of ELISA kits (0.001$40 \mathrm{ng} / \mathrm{mL}$ depending of the hormone targeted) assessing individual hormone quantification. However, our sensing approach has the advantage of multiplexing, a larger working range and a relatively low analysis time of $32 \mathrm{~min}$ per point. Moreover, each multiplex SPRi immunosensing surface showed good stability on which over 21 binding/regeneration cycles were performed.

Table 2 SPR sensing performance for a GNP-Ab $b_{2}$ multiplex immune assay for insulin, glucagon and somatostatin. The presented $\triangle R(\%)\left(C_{0}\right)$ is the sensor's response to the GNPs-Ab 2 conjugates.

\begin{tabular}{ccccc}
\hline Hormone & $\Delta \mathrm{R}(\%)\left(\mathrm{C}_{0}\right)$ & $\begin{array}{c}\text { LOD } \\
{[\mathrm{ng} / \mathrm{mL}]}\end{array}$ & $\begin{array}{c}\text { LOD } \\
{[\mathrm{nM}]}\end{array}$ & $\begin{array}{c}\text { Dynamic } \\
\text { Range } \\
{[\mathrm{ng} / \mathrm{mL}]}\end{array}$ \\
\hline Insulin & $1.32 \pm 0.03$ & 0.90 & 0.15 & $3.9-270$ \\
Glucagon & $1.40 \pm 0.03$ & 1.35 & 0.39 & $5.0-1,977$ \\
Somatostatin & $1.51 \pm 0.04$ & 2.00 & 1.22 & $6.6-4,000^{\mathrm{a}}$
\end{tabular}

a Highest concentration tested

The use of GNPs for SPR signal amplification led to a remarkable LOD improvement for all tested hormones. An increase of 9 fold for insulin, 10 fold for glucagon and 200 fold for somatostatin detection was obtained as compared to the multiplex sensing approach without using GNPs-conjugates amplification $^{22}$. Noteworthy is the fact that somatostatin showed a dramatic improvement in LOD. This could be explained by the fact that somatostatin is the smallest of the targeted hormones in this study ( $\mathrm{MW}=1637.88 \mathrm{Da})$. Thus, under the same immobilization conditions, the maximum amount of immobilization is expected to be lower than that of the higher MW hormones ${ }^{46}$. This could lead to less steric hindrance for binding of the large GNPs-Ab ${ }_{2}$ conjugates. This is corroborated by the fact that despite showing a similar response for primary antibody injection, somatostatin produced a slightly larger SPR shift for GNPs-Ab 2 conjugates (Fig. 5).

The LOD achieved in this study is in accordance with previous studies where detection of insulin ${ }^{15}$ and glucagon ${ }^{16}$ secreted from 10 islets was achieved at $15 \mathrm{mM}$ glucose. Therefore, the somatostatin secreted from this small population of islets could be effectively and accurately detected by our proposed approach, opening the possibility of gaining better understanding of its paracrine communications associated with abnormal islets' function in diabetes.

\section{Conclusions}

To address the challenges in detection of low molecular weight hormones secreted in very low concentration by human islets such as somatostatin, we investigated three GNPs amplification strategies using an SPRi-based biosensing approach. Although the amplification method involving the conjugation of primary antibodies with GNPs showed the best performance for sensing of individual hormones, it presented large cross-reactivity during multiplex experiments. This cross-reactivity was successfully circumvented using an immunoassay with secondary antibodies conjugated to GNPs as amplification. We successfully achieved multiplex detection of three pancreatic islet related hormones with an LOD of $0.15 \mathrm{nM}, 0.39 \mathrm{nM}$ and $1.22 \mathrm{nM}$ for insulin, glucagon and somatostatin respectively with a total analysis time of $32 \mathrm{~min}$ per point. This performance is comparable with previously reported detection sensitivity of insulin and glucagon secreted from 10 islets as well with the individual hormones sensing using conventional ELISA kits.

The possibility of working with a small pancreatic islet population combined with the advantage of multiplexing, a wide working concentration window and a low analysis time, makes our sensor very suitable for its future application in secretion-monitoring of the pancreatic islets, particularly for understanding the paracrine effect of somatostatin on glucoseinduced insulin and glucagon secretion. Furthermore, integrating a microfluidic perfusion platform with the proposed sensing approach could allow performing multiparametric analysis of an islets' SF in the context of discovery of novel drugs for diabetes treatment.

\section{Conflicts of interest}

There are no conflicts to declare

\section{Acknowledgments}

The authors acknowledge the financial support from the Natural Science and Engineering Research Council of Canada (NSERC) and the NSERC-CREATE in ISS grants, and FRC scholarship from Consejo Nacional de Ciencia y Teconología de México (CONACYT) and Secretaría de Educaión Pública de México (SEP). The authors are also thankful to Dr. F. Melaine for her help during the initial exploration of GNPs as amplification strategy.

\section{References}

1. F. R. Castiello, K. Heileman and M. Tabrizian, Lab on a Chip, 2016, 16, 409-431.

2. M. Braun, R. Ramracheya, S. Amisten, M. Bengtsson, Y. Moritoh, Q. Zhang, P. R. Johnson and P. Rorsman, Diabetologia, 2009, 52, 1566-1578.

3. S. J. Le Marchand and D. W. Piston, Journal of Biological Chemistry, 2010, 285, 14389-14398.

4. V. Grill, M. Gutniak, A. Roovete and S. Efendić, The Journal of Clinical Endocrinology \& Metabolism, 1984, 59, 293-297.

5. O. Segers, M. Vroede, Y. Michotte and G. Somers, Diabetic Medicine, 1989, 6, 232-238.

6. K. Ramachandran, X. Peng, K. Bokvist and L. Stehno-Bittel, Br. J. Pharmacol., 2014, 171, 3010-3022.

7. D. T. Nguyen, D. van Noort, I. K. Jeong and S. Park, Biofabrication, 2017, 9, 015021.

8. Y. Xing, M. Nourmohammadzadeh, J. E. M. Elias, M. W. Chan, Z. Q. Chen, J. J. McGarrigle, J. Oberholzer and Y. Wang, Biomed. Microdevices, 2016, 18, 9.

9. M. Braun, R. Ramracheya, P. R. Johnson and P. Rorsman, Annals of the New York Academy of Sciences, 2009, 1152, 187-193. 
10

S. T. Hanna, G. M. Pigeau, J. Galvanovskis, A. Clark, P. Rorsman and P. E. MacDonald, Pflug. Arch. Eur. J. Physiol., 2009, 457, 1343-1350.

11. D. S. Luciani and J. D. Johnson, Molecular and cellular endocrinology, 2005, 241, 88-98.

12. M. Braun, R. Ramracheya, M. Bengtsson, A. Clark, J. N. Walker, P. R. Johnson and P. Rorsman, Diabetes, 2010, 59, 1694-1701.

13. R. Ramracheya, C. Ward, M. Shigeto, J. N. Walker, S. Amisten, Q. Zhang, P. R. Johnson, P. Rorsman and M. Braun, Diabetes, 2010, 59, 2198-2208.

14. K. Terao, M. Gel, A. Okonogi, A. Fuke, T. Okitsu, T. Tada, T. Suzuki, S. Nagamatsu, M. Washizu and H. Kotera, Sci Rep, 2014, 4, 6 .

15. M. G. Roper, J. G. Shackman, G. M. Dahlgren and R. T. Kennedy, Analytical chemistry, 2003, 75, 4711-4717.

16. J. G. Shackman, K. R. Reid, C. E. Dugan and R. T. Kennedy, Anal. Bioanal. Chem., 2012, 402, 2797-2803.

17. A. R. Lomasney, L. Yi and M. G. Roper, Analytical Chemistry, 2013, 85, 7919-7925.

$18 . \quad$ J. P. Lafleur, A. Jonsson, S. Senkbeil and J. P. Kutter, Biosens. Bioelectron., 2016, 76, 213-233.

19 S. S. Hinman, K. S. McKeating and Q. Cheng, Analytical Chemistry, 2018, 90, 19-39.

20. G. Spoto and M. Minunni, The Journal of Physical Chemistry Letters, 2012, 3, 2682-2691.

21. H. J. Lee, D. Nedelkov and R. M. Corn, Analytical Chemistry, 2006, 78, 6504-6510.

22. F. R. Castiello and M. Tabrizian, Analytical Chemistry, 2018, 90, 3132-3139.

23. O. Cabrera, D. M. Berman, N. S. Kenyon, C. Ricordi, P. O. Berggrern and A. Caicedo, Proc. Natl. Acad. Sci. U. S. A., 2006, 103, 2334-2339.

$24 . \quad$ L. A. Lyon, M. D. Musick and M. J. Natan, Analytical Chemistry, 1998, 70, 5177-5183.

25. J. Jung, K. Na, J. Lee, K. W. Kim and J. Hyun, Anal. Chim. Acta, 2009, 651, 91-97.

26. S. Szunerits, J. Spadavecchia and R. Boukherroub, Reviews in Analytical Chemistry, 2014, 33, 153-164.

27. M. Frasconi, C. Tortolini, F. Botre and F. Mazzei, Analytical Chemistry, 2010, 82, 7335-7342.

28. J. S. Mitchell, Y. Wu, C. J. Cook and L. Main, Analytical Biochemistry, 2005, 343, 125-135.

29. J. S. Mitchell and T. E. Lowe, Biosensors and Bioelectronics, 2009, 24, 2177-2183.

30. S. W. Zeng, D. Baillargeat, H. P. Ho and K. T. Yong, Chem. Soc. Rev., 2014, 43, 3426-3452.

31. D. C. Kabiraz, K. Morita, K. Sakamoto and T. Kawaguchi, Talanta, 2017, 172, 1-7.

32. I. E. Sendroiu, L. K. Gifford, A. Lupták and R. M. Corn, Journal of the American Chemical Society, 2011, 133, 42714273.

33. J. U. Lee, A. H. Nguyen and Sang J. Sim, Biosensors and Bioelectronics, 2015, 74, 341-346.

34. K. V. Gobi, H. Iwasaka and N. Miura, Biosens. Bioelectron., 2007, 22, 1382-1389.

35. S. Takae, Y. Akiyama, Y. Yamasaki, Y. Nagasaki and K. Kataoka, Bioconjugate Chemistry, 2007, 18, 1241-1245.

36.
37.

38.
S. Taufik, A. Barfidokht, M. T. Alam, C. Jiang, S. G. Parker and J. J. Gooding, Journal of Electroanalytical Chemistry, 2016, 779, 229-235.

J. D. Driskell, C. A. Jones, S. M. Tompkins and R. A. Tripp, Analyst, 2011, 136, 3083-3090.

M. C. Estevez, J. Belenguer, S. Gomez-Montes, J. Miralles, A. M. Escuela, A. Montoya and L. M. Lechuga, Analyst, 2012, 137, 5659-5665.

T. Kawaguchi, D. R. Shankaran, S. J. Kim, K. Matsumoto, K. Toko and N. Miura, Sensors and Actuators B: Chemical, 2008, 133, 467-472.

D. Juncker, S. Bergeron, V. Laforte and H. Li, Current Opinion in Chemical Biology, 2014, 18, 29-37.

J. T. Dias, L. Lama, J. Gantelius and H. Andersson-Svahn, Nanoscale, 2016, 8, 8195-8201.

J. Martinez-Perdiguero, A. Retolaza, L. Bujanda and S. Merino, Talanta, 2014, 119, 492-497.

R. B. Schasfoort, Handbook of surface plasmon resonance, Royal Society of Chemistry, United Kingdom 2nd Edition edn., 2017.

H. Vaisocherova, E. Brynda and J. Homola, Anal. Bioanal. Chem., 2015, 407, 3927-3953.

J. Lahiri, L. Isaacs, J. Tien and G. M. Whitesides, Analytical chemistry, 1999, 71, 777-790. 